\title{
The Italian Chapter of the MOST Project: Specific Changes in the Organization of Headache Centres Can Help Improve the Patient Journey
}

\author{
Lorenzo Chiodo, ${ }^{1}$ Elisabetta Grillo, ${ }^{1}$ Elia Lahouiri, ${ }^{2}$ Marco Marchina, ${ }^{2}$ Marco Pedrazzoli ${ }^{2}$ and Matias Ferraris ${ }^{3}$ \\ 1. Novartis Farma SPA, Origgio, Varese, Italy; 2. LSC Lifesciences Consultants, Milan, Italy; 3. Novartis Pharma AG, Basel, Switzerland
}

DOl: https://doi.org/10.17925/USN.2021.17.1.48

ntroduction: Headache disorders are a major cause of disability, and their treatment poses a major burden for neurology clinics. The MOST project (Migraine network SoluTion) was a global programme that examined care pathways for patients with headache. Methods: The results of the 26 Italian centres involved in MOST were analysed. Data were collected in an interview-style process with healthcare professionals. Five aspects of the patient journey were analysed (patient referral, triage and allocation of priorities, time and resources used, role of the nurse and delegation of tasks, follow-up), and pitfalls and best practices were identified for each. Results: A total of 155 healthcare professionals were interviewed for data collection. Waiting times for first visit to centres ranged from 1 to 18 months (mean 5 months), while waiting times for follow-up visits ranged from a few days to up to 18 months (mean 4 months). Three types of networks were identified: centralized, unregulated multi-level and decentralized. Conclusion: Greater consistency in routine practice among specialized headache centres and more integration with primary care are important aspects that can improve waiting times and care of patients with headache disorders. Specialist referrals and involvement of neurology trainees are worthy of consideration. Nurses should have a greater role in patient education and organization of services.

\section{Keywords}

Headache, migraine, waiting lists, access, care, network, practice

Disclosures: Lorenzo Chiodo is an employee of Novartis Farma SpA. Elisabetta Grillo is an employee of Novartis Farma SpA. Elia Lahouiri was an employee of LSC Lifesciences Consultants, Milan, Italy, while the study was conducted; now Alira Health, Barcelona, Spain. Marco Marchina was an employee of LSC Lifesciences Consultants, Milan, Italy, while the study was conducted; now Bionest Partners, Paris, France. Marco Pedrazzoli is an employee of LSC Lifesciences Consultants, which has consulting projects with Novartis Pharma AG. Matias Ferraris is an employee of, and holds shares in, Novartis Pharma AG. The MOST project has been funded by Novartis Pharma AG and Novartis Farma SpA.

Acknowledgements: The authors thank Patrick Moore, who provided editorial assistance on behalf of Health Publishing \& Services Srl. This unconditional support was funded by Novartis Farma SpA.

Review process: Double-blind peer review.

Compliance with ethics: This article involves an assessment of interview questionnaires and does not contain any studies with human or animal subjects performed by any of the authors. Participants gave informed consent to voluntarily participate in the project. All participants had the right to withdraw from the interview at any time.

Authorship: The named authors meet the International Committee of Medical Journal Editors (ICMJE) criteria for authorship of this manuscript, take responsibility for the integrity of the work as a whole, and have given final approval for the version to be published.

Access: This article is freely accessible at touchNEUROLOGY.com (c) Touch Medical Media 2021

Received: 25 November 2020

Accepted: 4 May 2021

Published online: 22 July 2021

Citation: touchREVIEWS in Neurology. 2021;17(1):48-52

Corresponding author: Lorenzo Chiodo. Largo Umberto Boccioni, 121040 Origgio, Varese, Italy.

E: Iorenzo.chiodo@novartis.com

Support: The publication of this article was supported by Novartis Farma SpA.
In 2018, the Global Burden of Disease study placed headache disorders as the second leading cause of years lived with disability. ${ }^{1}$ As another indicator of its burden, headache was ranked among the leading causes of years lived with disability. ${ }^{1}$ Given its prevalence and severe symptom burden, headache has a consequential negative impact on the quality of life of many individuals. Indeed, a worldwide survey found that high proportions of patients with migraine report psychological issues, such as depression, difficulty in sleeping and fear of another attack, as well as problems in professional and social domains. ${ }^{2}$ The estimated direct annual costs of migraine alone in the USA are reported to be more than $\$ 17$ billion, although considering indirect costs, such as lost productivity and reduced quality of life, the total economic costs are likely to be much higher. ${ }^{3}$

Treatment of headache also poses a major burden for neurology clinics. In fact, it has been estimated that up to one-third of patients presenting to neurology clinics are seeking treatment for headache. ${ }^{4}$ Paradoxically, despite its negative impact, headache is both underdiagnosed and undertreated. ${ }^{5}$ In routine care, waiting lists for first consultation are often excessively lengthy; in the emergency setting, headache is responsible for up to $20 \%$ of complaints, ${ }^{6}$ leading some patients to leave the hospital before receiving treatment.? Long waiting lists are also a characteristic of routine care, with neurology clinics reporting times of up to a year or longer. ${ }^{8}$ Thus, taking the above into consideration, it is no understatement that the patient journey of individuals with headache is long and difficult. In one recent study from Brazil, headache started an average of 17 years before the first appointment with a headache specialist. ${ }^{9}$

In Europe, the care pathway for individuals with headache has been described to be 'a series of dead ends'. Many patients would benefit from medical care, but it is either unavailable or hard to access. ${ }^{10}$ In a survey carried out in Italy among patients with migraine, only $30 \%$ of patients reported that they were able to access treatment provided by specialist care centres. ${ }^{11}$ This demonstrates that even where headache services are present, they tend to be focused in specialist headache clinics, delivering high-end multidisciplinary care, but with very limited capacity, thereby generating long waiting lists. The patient journey is even more difficult in primary care, where many patients fail to receive a diagnosis, and where there is inadequate knowledge of the nature and mechanism of headaches, giving rise to suboptimal management.12

Given the limited resources available and with the aim of improving care and access to treatment of headache, assessment of current services is essential. This can help identify difficulties encountered by various centres, understand the reasons for long waiting lists, and identify best practice that can be shared with other centres and in other settings. Defining headache care quality indicators is, 
Figure 1: Distribution of waiting times for first visit at the 26 centres

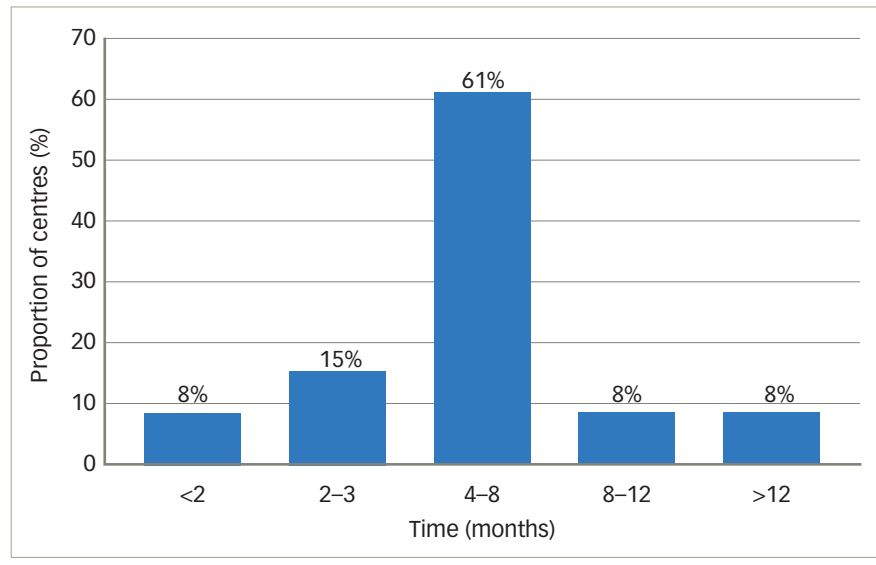

therefore, important to monitor and rate the overall quality of care with the objective of reducing the burden of headache and improve the quality of care received. ${ }^{13,14}$ With this aim in mind, the European Headache Federation (EHF) and Lifting The Burden (LTB) provisionally defined 'headache service quality' and have developed methods with which to measure it. ${ }^{15}$ In a previous survey in Italy, carried out at six specialist care centres, several indicators of the quality of care were identified. ${ }^{16}$ Moreover, the benchmark indicators were found to be adequate in judging the quality of care. In another study, using the joint definitions proposed, a survey of 14 European specialist care centres found that better standards were needed, and that it would be desirable to establish additional benchmarks to judge the quality of services offered. Accordingly, the EHF and LTB presented 10 roleand performance-defining standards that can be utilized by specialized headache centres to rate the quality of care with several indicators. ${ }^{17}$

Within the general framework of identifying critical factors in the patient's journey that can be improved upon, and thus improve the overall quality of care offered, the MOST (Migraine network SoluTion) was a global programme that examined the overall care pathways involved in management of patients with headache..$^{18}$ In particular, MOST evaluated the patient's journey, and the management and resources involved within selected networks (both general practitioners and specialized clinics). Critical issues in the patient journey were identified, such as difficulty accessing specialized care and long waiting lists. The programme had the overarching goal of providing appropriate intervention and sharing best practice to improve access to specialized care for patients with headache. Herein, the results of the Italian centres involved in the MOST project are presented.

\section{Materials and methods}

Seven countries (Italy, Spain, France, Ireland, Germany, Canada and Australia) participated in the MOST project. Data were collected in an interview-style process involving healthcare professionals (specialists, general practitioners, nurses and other professionals [e.g. pharmacists, psychologists]). The project investigated waiting times and how patient journeys varied across different healthcare networks, along with potential factors limiting access to care in these settings. Five aspects of the patient journey were analysed in detail, these included: i. patient referral; ii. triage and allocation of priorities; iii. time and resources used; iv. role of the nurse and delegation of tasks; and v. follow-up.

Four common types of biases in data gathering were proactively considered along with strategies to minimize bias. These included:
Figure 2: Distribution of waiting times for follow-up visits at the 26 centres

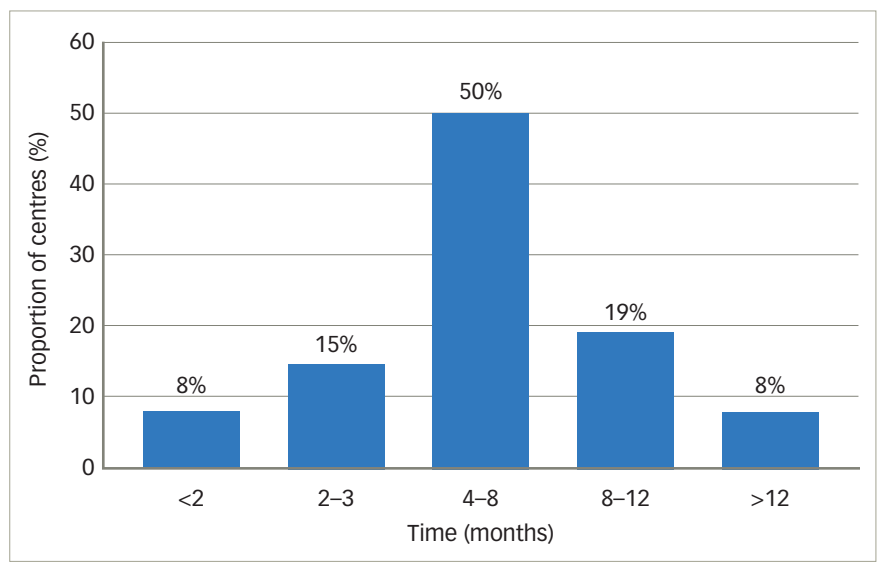

i. self-selection bias (i.e. centres could not select or invite themselves for inclusion, but rather a specific selection process was used); ii. singleresponder bias (interviewing multiple healthcare professionals within the same network reduces inconsistencies in the information collected); iii. single-interviewer bias (multiple interviewers were involved); iv. sponsor bias (interviewees were asked to not discuss any drugs marketed by the sponsoring pharmaceutical company).

\section{Results}

\section{Centres and healthcare professionals involved}

Across the seven countries, a total of 55 headache centres were involved, in which, 400 healthcare professionals manage over 50,000 patients. In Italy, 26 centres participated in the project. The various headache centres have a relatively homogeneous distribution throughout Italy. Data were collected in an interview-style process with 155 healthcare professionals (81 specialists, 18 general practitioners, 24 nurses and 32 other professionals). Each in-depth interview lasted 30-60 minutes.

\section{Waiting lists}

Waiting times for first visit to centres were highly variable, ranging from 1 to 18 months, with a mean of 5 months (Figure 1). No centre had a waiting list of $<1$ month. Two centres (8\%) had waiting lists of $<2$ months, and four centres had a waiting list of 2-3 months (15\%). Sixteen (61\%) had a waiting list of $4-8$ months. The remaining four centres reported a waiting list of $8-12(8 \% ; n=2)$ or $>12$ months $(8 \%$; $n=2)$.

Waiting times for follow-up visits were also quite variable, ranging from a few days to up to 18 months, with a mean of 4 months (Figure 2). Two centres had waiting lists of $<2$ months $(8 \%)$, four centres $(15 \%)$ had a waiting list of 2-3 months, and 13 (50\%) had a waiting list of 4-8 months. Five centres (19\%) had a waiting list of 8-12 months, and two centres (8\%) had a waiting list of $>12$ months. Some interviewees mentioned that waiting lists may change with the introduction of anti-calcitonin generelated peptide (CGRP) antibodies as antimigraine agents, although this was not clear at present.

\section{Types of centres}

Three types of networks were identified: centralized $(n=11)$, unregulated multi-level $(n=9)$ and decentralized $(n=6)$ (Figure 3). A centralized network is characterized by the presence of a reference centre that encourages referral from general practitioners ( $74 \%$ of patients). Access to the centre is not limited and patients are not required to have a previous neurological examination. The large number of second- and third-tier 
Figure 3: Types of headache centres identified

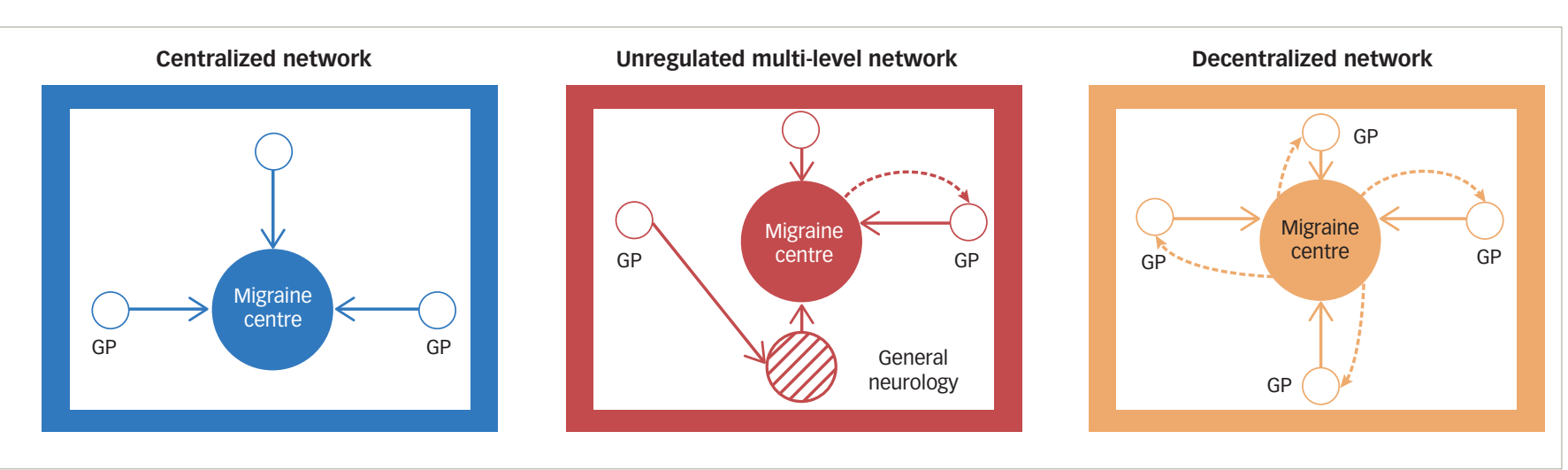

Circles identify patient access and referrals to migraine centres. Adapted with permission from Kainth et al., $2018 .{ }^{18}$ GP = general practitioner.

Figure 4: Pitfalls and best practices identified in the patient journey

\section{Pitfalls}
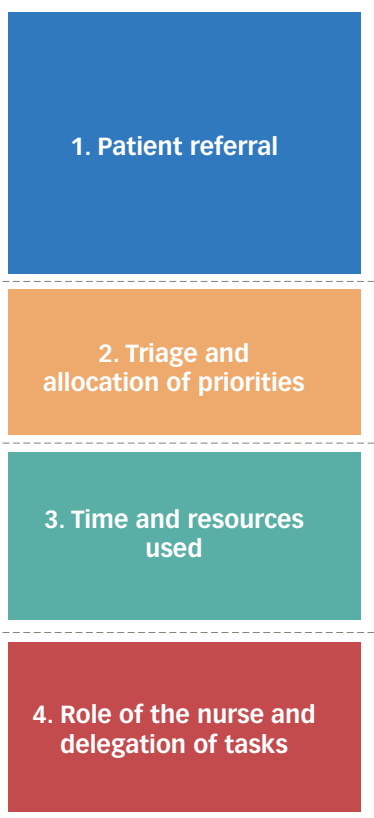

5. Follow-up and specialists
- General practitioners have limited knowledge of the available preventive therapies

- Lack of communication between general practitioners

- Some general practitioners refer all patients for a second diagnostic opinion

- Triage before patient access to centres is not available for most centres

- All patients are assigned the same visit slots despite individual history and severity of past disease

- Limited availability of dedicated nurses and interns at most centres - In $85 \%$ of centres, nurses are not involved in treatment
of patients

- Nurses usually have an administrative or educational role, and not an active role in patient care

- General practitioners have limited experience in treatment and follow-up of patients with migraine

\section{Best practice}

- Letter sent to general practitioners to communicate access criteria for patients with headache

- Organization of a training course for general practitioners to learn about treatment of headaches

- Regional treatment pathway for patients with migraine

- Access to specialized clinics allowed only by request from specialists (and not directly by general practitioners)

- Triage is carried out by interns before patients enter the clinic

- $80 \%$ of patients referred to the headache centre for diagnosis and treatment have chronic migraine

- First visit is in an outpatient clinic managed by an intern; nurses assist in patient visits, prepare patients for treatment and provide education

- A dedicated day hospital is organized for further tests and to support patients with severe migraine

- Nursing staff prepare botulinum toxin for treatment

- Use of a headache diary after the first specialist visit

- $90 \%$ of all follow-up visits are delegated to the general practitioner after education is provided patients causes a substantial increase in waiting times. The general neurologist has a limited role in the management of less severe patients. In some of these centres, problems with triage and data collection were noted at first visit given the long waiting times. During follow-up, $90 \%$ of patients were managed by the centre except for more severe cases.

In an unregulated multi-level network, the headache centre was third-level care, recognized for management of headache at a regional or even national level. The general neurologist acted as a filter for patients who were stable and less severe. Patients managed by these highly specialized centres often had a long history of headache and were refractory to previous treatment. Considering waiting lists for a first visit, some centres noted problems in establishing a shared treatment plan. Most of these patients were managed by the centre during follow-up.
In a decentralized network, the headache centre prioritizes referral from general practitioners, and patients are sent to the centre for diagnostic examinations and to initiate preventive treatment. Around half of patients $(51 \%)$ were managed by the centre after initiation of therapy, and the other half were managed by the patient's general practitioner after definition/initiation of therapy. If changes to therapy were needed, the general practitioner sent the patient back to the headache centre for consultation. Some of these centres noted that there were problems in establishing a standard document for follow-up.

\section{Patient journey}

After detailed analysis of each aspect of patient journeys, some recurring pitfalls and best practices have been identified (Figure 4). For patient referral, given the limited knowledge of headache management by general practitioners and lack of communication with specialists, it was 
thought that greater communication between general practitioners and specialists should be encouraged. For triage, healthcare professionals considered best practice to have neurology trainees perform this prior to entering the clinic. It was further noted that there was limited availability of dedicated nurses and neurology trainees at most centres. To overcome this, the best practice identified entailed a first visit by both neurology trainee and a nurse, with the latter assisting patients and providing education. Moreover, dedicated day hospital assistance can be organized for further tests and to support patients with severe headache. Lastly, considering the overall lack of experience in follow-up by general practitioners, it was believed that they could carry out follow-up after adequate education is provided. This would help to optimize therapy and reduce waiting lists.

\section{Discussion}

In a previous study involving 28 headache centres across Europe, the average waiting time for first consultation was 6 months, with peaks even beyond 12 months. ${ }^{18}$ The results of the present analysis found a similar spectrum of waiting times across Italy. In a previous study in Italy, while waiting times were not documented among the different centres, all noted that a formal triage system was, however, in place. ${ }^{16}$ Long waiting lists are one of the main factors that negatively impact access to care. ${ }^{19}$ Accordingly, optimization of waiting lists can thus dramatically improve access to care after adequate identification of barriers.

Five areas were identified in a prior analysis by Kainth et al., which were also evaluated in the present analysis. ${ }^{18}$ In that study, each of the five areas was affected by barriers that negatively influenced the patient journey: i. inappropriate referrals for low-frequency episodic migraine or those under acute treatment; ii. limited availability of triage; iii. limited resources dedicated to migraine; iv. inadequate delegation of activities; and v. suboptimal organization of follow-up. Herein, we further explored each of these areas and propose specific solutions to overcome barriers specifically linked to each area.

One of the aspects identified and related to overload of specialized headache centres was the lack of experience in treating and following patients by general practitioners. To overcome this, educational courses could be organized to foster better communication between specialists and general practitioners, as well as pharmacists. Especially during follow-up, with adequate training, general practitioners could manage and follow the majority of patients once a treatment plan has been established. Moreover, pharmacists could be educated so that they can play a more prominent role in referring the most severe patients to specialized headache clinics. These implementations would have the benefit of dramatically reducing waiting times for both initial visits and follow-up, when needed, as it was recognized that while the majority of patients could be effectively followed by general practitioners, a proportion will still need to be followed by specialist care. This would also allow for more patients to overcome some of the barriers identified for successful care, by having greater access to specialist care, receive an accurate diagnosis, and obtain adequate acute and preventive treatment. ${ }^{19}$

With regard to triage, the best practice identified entailed the use of interns to perform this prior to presentation to a specialized clinic. In this way, patients can carry out the first specialist visit with more clinical information, as diagnostic examinations could be performed earlier. These visits, possibly in an ambulatory setting, could also be made in parallel with a nurse who can provide education about headache, explain the use of a headache diary, and prepare treatments. This would help to optimize time and resources, while encouraging greater collaboration and communication between different healthcare professionals.

A three-tier approach to patient management has been recommended to improve efficiency of care that involves general primary care, special interest headache care, and specialist centres. ${ }^{17,20}$ Three types of centre were identified in the present analysis, which all have some common elements with the three-tier approach, although each was organized differently. Some shortcomings were identified in each type of centre. For instance, centralized networks tended to have long waiting times, which is related to unlimited access and no requirements for prior neurological examination. As mentioned, these aspects could be optimized by making prior visits with a neurology trainee mandatory so that diagnostic examinations are carried out beforehand. In addition, for unregulated multi-level and decentralized networks, standardized treatment plans can be shared with all healthcare professionals involved in the patient's management, which would also allow patients to be followed in primary care. Overall, the best practices identified provide specific guidance on how to improve the level of care and overall access, which are largely in line with the suggested standards and criteria published previously. ${ }^{17,20}$

Another area that warrants comment is the possible role of digital systems to prioritize and optimize waiting lists according to the needs of the individual patient and ensure better preparation for first visit. It would be relatively simple to include information about priority for initial and follow-up visits, and nurses with an administrative role could be very useful in scheduling visits based upon specific information provided by the prescribing physician. This would streamline waiting lists further. In this regard, the current COVID-19 pandemic may provide the opportunity to promote digitalized patient management and optimize patient visits, given the additional impact of the pandemic on healthcare services in general. Lastly, it should be mentioned that new tactics and solutions need to be implemented in light of forthcoming anti-CGRP therapies, considering that patients may need training for self-injection and follow-up schedules will need to be defined.

Our study has some limitations. First, not all headache centres in Italy were included, and thus there may be some selection bias. Analysis of actual waiting lists would be preferred to minimize bias. Data were collected from interviews, which may also be subject to bias, and we had no means to objectively validate the responses. Patient perceptions were also not taken into account in the treatment journey, and it would be worthwhile to assess these aspects to further improve the standard of care. Lastly, there was no form of statistical analysis; therefore, the results and conclusions could not be quantified.

Based on our results, greater consistency in routine practice among specialized headache centres, independently of the types of organization of the centre, and more integration with primary care are important aspects that can improve waiting lists and improve care of patients with headache disorders. Specialist referrals and better preparation for initial visits through use of neurology trainees in ambulatory settings are also worthy of consideration. Lastly, nurses will also play a primary role in patient education and organization of services that will undoubtedly involve greater use of digital platforms, remote technology, and smartphone apps to monitor patients and make sure they are fully prepared for initial and follow-up visits. $]$ 


\section{Original Research Headache Disorders}

Saylor D, Steiner TJ. The global burden of headache. Semin Neurol. 2018;38:182-90.

2. Martelletti P, Schwedt TJ, Lanteri-Minet M, et al. My Migraine Voice survey: a global study of disease burden among individuals with migraine for whom preventive treatments have failed. J Headache Pain. 2018;19:115.

3. Goldberg LD. The cost of migraine and its treatment. Am J Manag Care. 2005;11(2 Suppl.):S62-7.

4. World Health Organization. Headache disorders. 2016. Available at: www.who.int/en/news-room/fact-sheets/detail/ headache-disorders (accessed 7 May 2021).

5. Moriarty $\mathrm{M}$, Mallick-Searle T. Diagnosis and treatment for chronic migraine. Nurse Pract. 2016;41:18-32.

6. Royl G, Ploner CJ, Mockel M, et al. Neurological chief complaints in an emergency room. [Article in German]. Nervenarzt. 2010;81:1226-30.

7. Hoyer C, Stein P, Alonso A, et al. Uncompleted emergency department care and discharge against medical advice in patients with neurological complaints: a chart review. BMC Emerg Med. 2019;19:52

8. Irish Health. Big jump in neurology waiting list. 2014 Available at: www.irishhealth. com/article. html?id $=24245$ (accessed 7 May 2021)

9. Peres MFP Swerts DB, de Oliveira AB, et al. Migraine patients' journey until a tertiary headache center: an observational study. J Headache Pain. 2019:20:88

10. Leonardi M, Ruiz De La Torre E, Steiner TJ, et al. Value of treatment of headache patients and need to improve headache patients' journey. Neurol Sci. 2018;39(Suppl. 1):115-6.

11. CENSIS. Vivere con I'emicrania: Dalla sottovalutazione a condizionamento esistenziale [Living with migraine: From underestimation to existential conditioning. Rome, Italy: Solari grafiche srls, 2019. Available at: www.censis.it/sites/default/files/downloads/Sintesi_2019. pdf (accessed 15 June 2021)

12. Davies PTG, Lane RJM, Astbury T, et al. The long and winding road: the journey taken by headache sufferers in search of help. Prim Health Care Res Dev. 2019;20:e4.

13. Peters $\mathrm{M}$, Jenkinson $\mathrm{C}$, Perera S, et al. Quality in the provision of headache care. 2: defining quality and its indicators. J Headache Pain. 2012;13:449-57.

14. Peters M, Perera S, Loder E, et al. Quality in the provision of headache care. 1: systematic review of the literature and commentary. J Headache Pain. 2012:13:437-47.
15. Schramm S, Uluduz D, Gouveia RG, et al. Headache service quality: evaluation of quality indicators in 14 specialist-care centres. J Headache Pain. 2016;17:111

16. Pellesi L, Benemei S, Favoni V, et al. Quality indicators in headache care: an implementation study in six Italian headache care: an implementation study in six Italian

17. Steiner $T J$, Gobel $H$, Jensen $R$, et al. Headache service quality: the role of specialized headache centres within structured headache services, and suggested standards and criteria as centres of excellence. J Headache Pain. 2019;20:24.

18. Kainth P, Murphy N, Talabardon JN, et al. Management of migraine and the accessibility of specialist care - findings from a multi-national assessment of 28 healthcare networks. Eur Neurol Review. 2018;12:103-10.

19. Dodick DW, Loder EW, Manack Adams A, et al.

Assessing barriers to chronic migraine consultation, diagnosis, and treatment: results from the chronic migraine epidemiology and outcomes (CaMEO) study. Headache. 2016;56:821-34.

20. Steiner TJ, Antonaci F, Jensen R, et al. Recommendations for headache service organisation and delivery in Europe. I Headache Pain. 2011:12:419-26. 